\title{
BMJ Open Physical activity interventions for adults who are visually impaired: a systematic review and meta-analysis
}

\author{
Joanna Sweeting (1) ,1,2 Dafna Merom (1) , ${ }^{3}$ Putu Ayu Swandewi Astuti (D) ,2,4 \\ Michael Antoun, ${ }^{5}$ Kate Edwards (D) , ${ }^{1,6}$ Ding Ding (D) ${ }^{1,2}$
}

To cite: Sweeting J, Merom D, Astuti PAS, et al. Physical activity interventions for adults who are visually impaired: a systematic review and meta-analysis. BMJ Open 2020;10:e034036. doi:10.1136/ bmjopen-2019-034036

- Prepublication history and additional material for this paper are available online. To view these files, please visit the journal online (http://dx.doi. org/10.1136/bmjopen-2019034036).

Received 04 September 2019 Revised 28 December 2019 Accepted 10 January 2020

Check for updates

(c) Author(s) (or their employer(s)) 2020. Re-use permitted under CC BY-NC. No commercial re-use. See rights and permissions. Published by BMJ.

For numbered affiliations see end of article.

\section{Correspondence to}

Dr Joanna Sweeting;

joanna.sweeting@sydney.edu. $\mathrm{au}$

\section{ABSTRACT}

Objectives Compared with sighted individuals, people with visual impairment have a higher prevalence of chronic conditions and lower levels of physical activity. This review aims to systematically review physical activity interventions for those with a visual impairment and to assess their effectiveness.

Design A systematic review of articles reporting physical activity interventions in visually impaired individuals was conducted. Medline, EMBASE, The Cochrane Library, Cumulative Index to Nursing and Allied Health Literature, SPORTDiscus and the Physiotherapy Evidence Database were searched in August 2018. Meta-analyses were conducted on randomised controlled trials with the same outcome measure.

Setting Most interventions were conducted in a group setting, with some including an at-home, self-directed component.

\section{Participants Following identification of a recent} systematic review of physical activity interventions in children, our review focused on adults aged 18 years and older with a visual impairment.

Primary and secondary outcome measures Outcomes included measures of balance, mobility, mental well-being (eg, quality of life), number of falls, muscle strength, flexibility and gait.

Results Eighteen papers from 17 studies met inclusion criteria. Physical activity components include falls prevention and/or balance-based activities, walking, tai chi, Alexander Technique, Yoga, dance, aerobics and core stability training. Significant results in favour of the intervention were reported most commonly in measures of functional capacity (9/17 studies) and in falls/balancerelated outcomes (7/13 studies). The studies identified were generally small and diverse in study design, and risk of bias was high across several categories for most studies. Meta-analyses indicated non-significant effects of the included interventions on the Timed Up and Go, Chair Sit Test and Berg Balance Scale.

Conclusions Physical activity interventions in individuals with visual impairment incorporating activities such as tai chi, Yoga and dance can have positive results, particularly in physical measures such as mobility and balance. However, when performing a meta-analysis of randomised controlled trials, the evidence for effectiveness is less clear. More studies with larger sample sizes, stronger designs and longer follow-up periods are needed. PROSPERO registration number CRD42018103638.

\section{Strengths and limitations of this study}

This systematic review was registered a priori and conducted in line with two guidelines, Preferred Reporting Items for Systematic Reviews and Meta-Analysis and A Measurement Tool to Assess Systematic Reviews

- Six databases were used and a back-reference search of all included studies was conducted, with no limit on language or year of publication imposed.

- Risk of bias analysis was conducted independently by two reviewers using the validated Cochrane Collaboration tool

- A lack in common outcome measures allowed inclusion of only four studies in the meta-analysis.

\section{INTRODUCTION}

Physical activity is well established as a prophylactic for many non-communicable diseases including cardiovascular disease, certain cancers, hypertension and type 2 diabetes. ${ }^{1}$ In addition to physical health, regular physical activity is also known to benefit psychological well-being including a reduction in the risk of depression and anxiety, lowering of stress levels and improving mood. ${ }^{34}$ The Centers for Disease Control and Prevention (CDC) recommends at least $150 \mathrm{~min}$ per week of moderate intensity aerobic physical activity (or equivalent vigorous activity) for adults (aged 18-64). ${ }^{5}$ However, with the global prevalence of insufficient physical activity at nearly $30 \%$ in 2016 , it is imperative that regular physical activity continues to be promoted and encouraged worldwide. ${ }^{6}$ This is important not only in healthy populations, but also in those with diseases and conditions, such as cardiovascular disease and disabilities such as visual impairment. As highlighted by the CDC in the current physical activity guidelines, there is strong evidence that regular physical activity conveys important health benefits for individuals with a disability. ${ }^{5}$ However, adults with disabilities are three 
times more likely to have chronic conditions such as heart disease, diabetes and cancer, and nearly $50 \%$ of adults with a disability undertake no leisure time physical activity. ${ }^{6}$ More research among those with specific disabilities is needed to address these gaps and improve health outcomes for those with a disability. ${ }^{7}$

In the USA, the five most common functional disabilities are in mobility, cognition, independent living, hearing and vision. ${ }^{8}$ In 2015, an estimated 36 million $(0.49 \%$ of the total population, visual acuity worse than $3 / 60)$ people worldwide were blind, 217 million $(2.95 \%)$ had moderate or severe vision impairment (visual acuity worse than $6 / 18$ and $6 / 60$, respectively) and another 189 million $(2.57 \%)$ had mild vision impairment (visual acuity worse than 6/12). The most common causes of vision impairment include uncorrected refractive errors, cataracts, age-related macular degeneration, glaucoma and diabetic retinopathy. ${ }^{9}$

Visual impairment has been shown to detrimentally impact quality of life ${ }^{10} 11$ and to be associated with depression. ${ }^{12}$ Also concerning is the fact that studies have shown a higher mortality rate for visually impaired individuals compared with their sighted counterparts, although the underlying reasons are uncertain. ${ }^{13} 14$ Even at the mild end of the impairment spectrum, loss of vision can affect health and well-being, for example, through restriction of driving, potentially impacting an individual's sense of autonomy and freedom. ${ }^{15}$ Vision impairment has also been shown to be associated with less time spent in moderate-vigorous physical activity in the range of $26 \%-48 \%$ compared with sighted individuals. ${ }^{16-18}$ One potential reason for this discrepancy is the fear of falling associated with loss of vision and consequent poor balance. ${ }^{1617}$ For those able to navigate their local environment with the assistance of a guide dog or cane, physical barriers such as uneven, slippery or blocked footpaths can make it difficult to perform adequate physical activity. ${ }^{19}$ With the adverse effects that visual impairment can have on well-being, and extra challenges those with visual impairments face, it is of upmost importance that physical activity is encouraged in this population, given its beneficial impact on health and well-being.

To date, few interventions have included participants with vision impairment. In fact, it is more often the case that visual impairment or blindness are exclusion factors from physical activity interventions. With increasing recognition of the health disparities experienced by people living with disabilities and the lack of research by contrast,${ }^{20}$ it is important to ensure that the principle of inclusiveness is applied so that interventions are designed for those with disabilities. This review aims to systematically review physical activity interventions for those with vision impairment and to assess the effectiveness of the interventions in improving healthrelated (physical and mental) outcomes and issues encountered.

\section{METHODS}

\section{Eligible studies}

This systematic review included peer-reviewed articles reporting on physical activity interventions in visually impaired individuals. The research questions, search strategy and inclusion/exclusion criteria were determined prior to commencing the search. Although the initial research plan was to review studies across all age categories, the population of interest was restricted to adults (aged 18 years and over) following the initial search as a recent systematic review among children and adolescents was identified. ${ }^{21}$ We included experimental studies focusing on a physical activity intervention or those examining interventions with a clear physical activity component. Controls included individuals not exposed to the intervention or the baseline measurements of participants prior to commencement of the intervention (prepost study design). Both randomised controlled trials (RCTs) and non-randomised studies of interventions, including pre-post studies without a comparison group, were included to provide a more complete picture of all the studies in the literature, given the small number expected. Observational studies, reviews, case reports, abstracts, commentaries or other opinion pieces were excluded. No limit on publication date or language of publication was set to ensure broad coverage of the literature. Outcome measures included a range of physical measurements, such as body fat percentage, blood pressure, body mass, waist circumference; physical activity/ fitness measures such as flexibility, daily step count, balance and muscle strength and endurance; and wellbeing measures including social and emotional wellbeing and depression.

\section{Sources and search strategy}

We searched Medline (1946-August 2018), EMBASE (1947-August 2018), The Cochrane Library (1993August 2018), Cumulative Index to Nursing and Allied Health Literature (1982-August 2018), SPORTDiscus (1892-August 2018) and the Physiotherapy Evidence Database (1929-August 2018). Back references of all papers included in the review were also searched to identify additional articles. Search terms included those related to blindness and visual impairment (eg, vision disorders, visually impaired person and glaucoma) and physical activity (eg, exercise, sports, muscle strength, gait, dancing and rehabilitation). A targeted search of the Journal of Visual Impairment and Blindness was conducted due to indexing issues discovered during the backreference search. One article was discovered through this additional search. The final search strategy for Medline is outlined in online supplementary material 1 . This search strategy was adapted for use with the other bibliographic databases in combination with database-specific filters. An initial screen of all abstracts was conducted to identify potentially relevant studies (MA and JS). These studies were then simultaneously and independently reviewed by two reviewers (DD and PASA) to determine eligibility 
for inclusion in this review, with a third reviewer (DM) enlisted in the case of disagreement.

\section{Data collection}

Data were extracted from the eligible papers by JS and summarised into an Excel (Microsoft Office 365) spreadsheet with the following headings: Author, Year of Publication, Population (including age) and Setting, Visual Conditions, Exclusion Criteria, Study Design, Control Group, Theory (behind the intervention), Type of Physical Activity Intervention, Dose of Intervention (times per week, duration), Delivery (who delivered the intervention), Outcomes, Process Evaluation (eg, participation, adherence, drop out, feedback), Results, Other Notes and Funding Sources. We further condensed the extracted data under the headings seen in tables 1 and 2. Data extraction was checked by KE with agreement achieved on all studies through discussion. Data for one paper written in Farsi was extracted by a collaborator fluent in Farsi.

\section{Analysis}

The main characteristics and findings of each study were summarised and tabulated to provide an overview of the literature to date in this area. Where measures were common across RCT studies, a meta-analysis was conducted, using R Foundation for Statistical Computing V.3.6.0 to estimate the standardised mean difference and 95\% CI to assess the effectiveness of the interventions. The $\mathrm{I}^{2}$ was calculated as a measure of heterogeneity between studies. This review was conducted in line with the Preferred Reporting Items for Systematic Reviews and Meta-Analysis (PRISMA) ${ }^{22}$ and A Measurement Tool to Assess Systematic Reviews (AMSTAR2) guidelines $^{23}$ (online supplementary material 2 and 3). Risk of bias assessment was performed by JS and PASA, using the Cochrane Collaboration's tool for assessing risk of bias. This tool was used to rate RCTs with a low, high or uncertain risk of bias across six criteria including randomisation, allocation concealment, performance bias, ascertainment bias, incomplete outcome data and selective reporting. ${ }^{24}$ For non-randomised studies we considered the risk of bias due to incomplete data and selective reporting.

\section{Patient and public involvement}

Neither patients nor the public were involved in the design, conduct, reporting or dissemination of this research.

\section{RESULTS}

\section{Study selection}

A total of 10112 records were returned, with 6517 unique record titles and abstracts screened for possible inclusion. Of these, 56 full texts were obtained and reviewed, with 18 papers (from 17 studies) meeting the inclusion criteria (figure 1). Primary reasons for exclusion were the studies were conducted in children under the age of $18(n=19)$, were not reporting results of a trial of an intervention (eg, protocol papers; $\mathrm{n}=14$ ) and did not include physical activity as a key component of the intervention $(n=5)$. The studies were predominately funded by Government and/or Research Grants ( $n=9)$, with funding sources not specified by six studies. One intervention which examined the impact of the Alexander technique 2526 was funded by private sources including The Australian Society of Teachers of the Alexander Technique and the FM Alexander Trust (UK), in addition to government and research funding. One intervention was not sponsored.

\section{Study characteristics}

The characteristics of the studies are shown in online supplementary material 4 and summarised in table 1 . Most of the papers $(n=14,78 \%)$ were published in the 10 years preceding the date of the search (2008-2018). Nine employed an RCT study design, with the remaining eight studies using a pre-post format. Seven interventions were conducted in the USA with the remaining studies conducted in Europe $(n=5)$, Asia $(n=3)$ and Oceania $(n=2)$. Except for one study that was published in Farsi, all studies were published in English.

\section{Participants}

There was a total of 906 participants across the 17 interventions, with a mean of 53 and a median of 29 per study. The number of participants per study ranged from three to 391 with 14 studies consisting of 50 or fewer participants. The mean age across all studies was 62 years, with only two ${ }^{27} 28$ examining populations aged younger than 35 years. Approximately two-thirds (70\%) of participants were female, with three studies only including women. ${ }^{27} 2930$ Participants were recruited through a combination of local advocacy groups, community centre listings and by word-of-mouth in four studies. In eight studies participants were recruited from medical institutions such as hospitals, clinics, private practices and rehabilitation services. In two studies participants were recruited from residential care homes and one recruited participants from a university (recruitment method unknown in two studies).

Visual impairment was defined in several ways with varying levels of detail. Most studies provided cut-points of visual acuity (eg, 6/24 or worse), while some linked these cut points to those designated by the WHO International Classification of Diseases Codes. ${ }^{31} 32$ Visual conditions identified included age-related conditions such as macular degeneration and cataracts, diabetic retinopathy, glaucoma, corneal scars and congenital blindness.

\section{Intervention types}

Seven studies employed specific falls prevention and/or balance-based physical activity interventions. Of these, three used the Otago exercise programme ${ }^{30} 3334$ three used general physical activity training programme aiming to improve balance ${ }^{293536}$ and one used the Matter of 


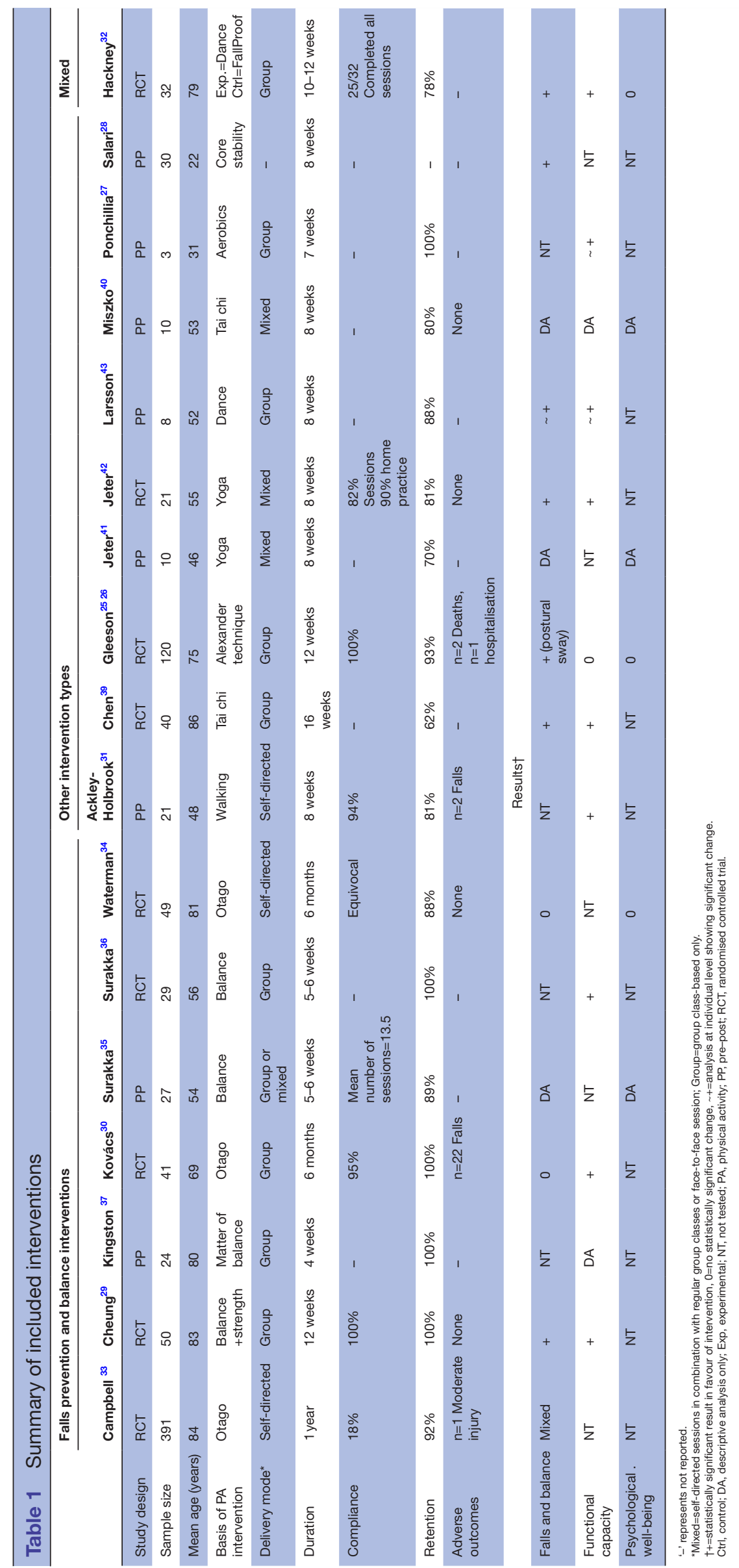

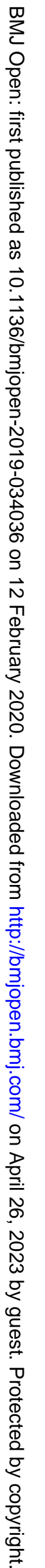



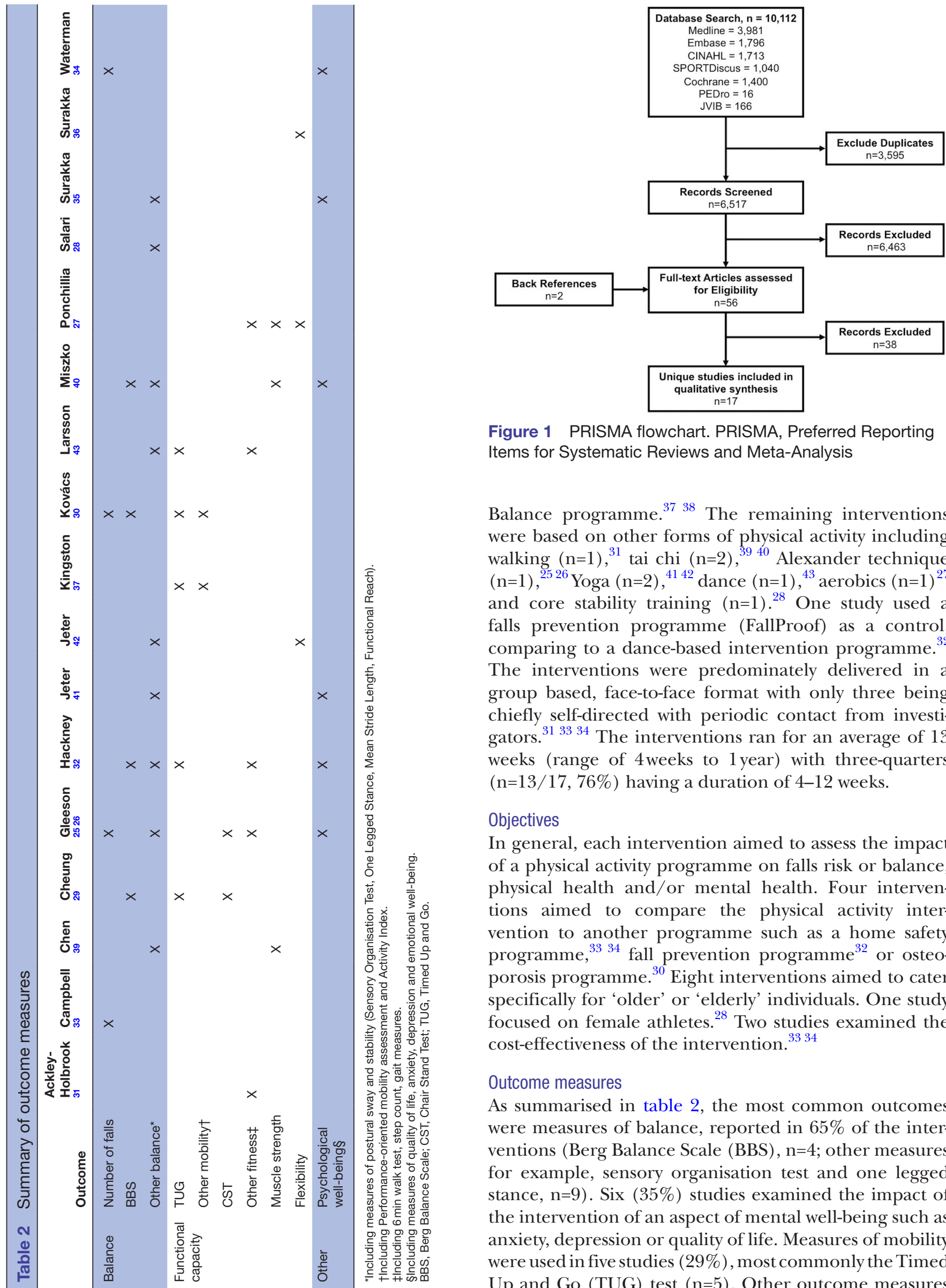

Figure 1 PRISMA flowchart. PRISMA, Preferred Reporting Items for Systematic Reviews and Meta-Analysis

Balance programme. ${ }^{37} 38$ The remaining interventions were based on other forms of physical activity including walking $(\mathrm{n}=1),{ }^{31}$ tai chi $(\mathrm{n}=2),{ }^{39}{ }^{40}$ Alexander technique $(\mathrm{n}=1),{ }^{25}{ }^{26}$ Yoga $(\mathrm{n}=2),{ }^{41}{ }^{42}$ dance $(\mathrm{n}=1),{ }^{43}$ aerobics $(\mathrm{n}=1){ }^{27}$ and core stability training $(n=1) .{ }^{28}$ One study used a falls prevention programme (FallProof) as a control, comparing to a dance-based intervention programme. ${ }^{32}$ The interventions were predominately delivered in a group based, face-to-face format with only three being chiefly self-directed with periodic contact from investigators. ${ }^{3134}$ The interventions ran for an average of 13 weeks (range of 4 weeks to 1 year) with three-quarters $(n=13 / 17,76 \%)$ having a duration of 4-12 weeks.

\section{Objectives}

In general, each intervention aimed to assess the impact of a physical activity programme on falls risk or balance, physical health and/or mental health. Four interventions aimed to compare the physical activity intervention to another programme such as a home safety programme,$^{33}{ }^{34}$ fall prevention programme ${ }^{32}$ or osteoporosis programme.$^{30}$ Eight interventions aimed to cater specifically for 'older' or 'elderly' individuals. One study focused on female athletes. ${ }^{28}$ Two studies examined the cost-effectiveness of the intervention. ${ }^{33} 34$

\section{Outcome measures}

As summarised in table 2, the most common outcomes were measures of balance, reported in $65 \%$ of the interventions (Berg Balance Scale (BBS), $n=4$; other measures for example, sensory organisation test and one legged stance, $n=9$ ). Six (35\%) studies examined the impact of the intervention of an aspect of mental well-being such as anxiety, depression or quality of life. Measures of mobility were used in five studies (29\%), most commonly the Timed Up and Go (TUG) test $(n=5)$. Other outcome measures 
included number of falls $(n=4)$, muscle strength $(n=3)$, flexibility $(n=3)$, gait $(n=3)$, anthropometric measures $(n=3)$, the chair stand test $(n=2)$ and sleep $(n=1)$.

\section{Synthesis of results}

Falls or balance-related outcomes were measured in 13 studies, with statistically significant results in favour of the intervention observed in seven (54\%) studies. Although more falls were recorded in the intervention group in the Campbell $e t a l^{33}$ study, further analysis showed fewer falls with increasing adherence to the exercise programme $(\mathrm{p}=0.001)$. Measures of functional capacity were used in 12 studies, with statistically significant results in favour of the intervention observed in nine $(75 \%)$ studies. Psychological well-being was measured in only six studies and no significant results were observed in these outcomes. No paper reported negative results that would suggest the intervention was detrimental to any aspect of health measured. Dropout reasons across all studies included medical problems $(n=10)$, lack of transport or travel time $(\mathrm{n}=7)$, dissatisfaction with programme $(\mathrm{n}=1)$, time $(\mathrm{n}=2)$ and other $(\mathrm{n}=3)$.

\section{Effectiveness of interventions on physical activity outcomes}

A meta-analysis was conducted where outcome measures were common across RCT interventions, namely for the TUG, Chair Sit Test (CST) and BBS. Only four studies were able to be included in the meta-analysis and the results are shown in figure 2. In all instances, the combined results crossed the line of null effect (mean difference $=0$ ) indicating non-significant effects of the interventions on each of the outcome measures. In addition, the wide $95 \%$ CIs indicate imprecision across the studies, potentially due to small sample sizes. Heterogeneity was low for the TUG and CST ( $\mathrm{I}^{2}=0 \%$ and $22 \%$, respectively) suggesting consistent null findings; however, it was high for the BBS $\left(I^{2}=53 \%\right)$.

\section{Study quality—risk of bias assessment}

The results of the risk of bias assessment can be found in table 3. In general, the RCTs showed a low risk of bias in randomisation ( $\mathrm{n}=6 / 9$ 'low') and allocation concealment $(n=6 / 9$ 'low'). Risk of attrition bias due to incomplete data was also low in the majority of all studies $(n=11 / 17)$. Of note, all but one study was categorised as 'unclear' or 'high' risk of reporting bias due to selective outcome reporting. In all pre-post studies it was not possible to determine if all outcomes were reported due to the lack of study protocol or registrations. Of the nine RCTs, study protocols were unavailable for five, two were missing a priori secondary outcomes, one followed the study protocol and one deviated from the study protocol.

\section{DISCUSSION}

This systematic review sought to summarise the effects of physical activity interventions in adults who are visually impaired. Based on the studies identified, there is
A

Timed up-and-go, seconds

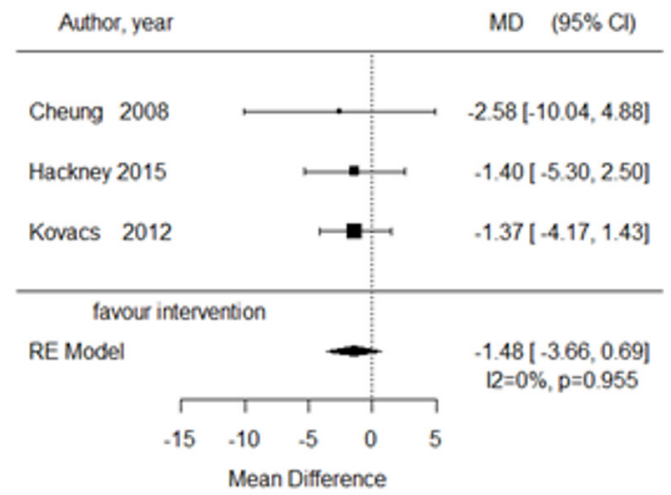

B

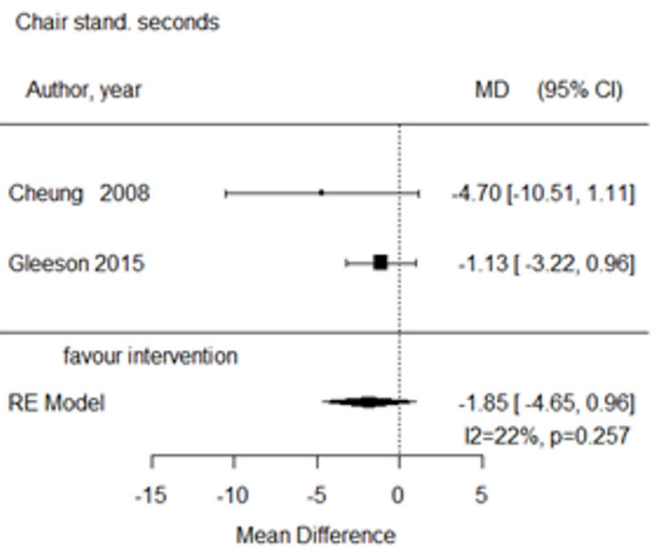

$\mathrm{C}$

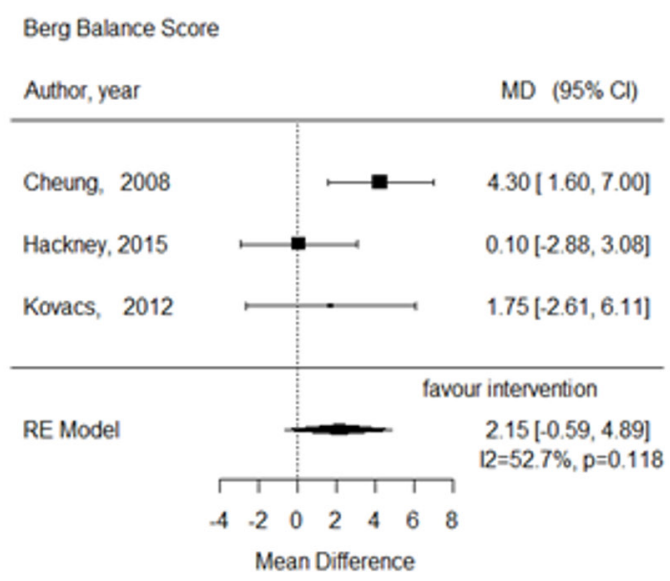

A. Timed Up and Go. B . Chair Stand Test. C. Berg Balance Scale Figure 2 Forest plots from meta analyses. (A) TUG. (B) CST (C) BBS. BBS, Berg Balance Scale; CST, Chair Stand Test; $M D$, mean difference; RE, random effects; TUG Timed Up and Go.

evidence that physical activity interventions are beneficial to adults with visual impairment, with positive health benefits observed particularly in outcomes related to functional capacity. However, when focusing on RCTs, where the risk of bias is lower, and examining combined 
Table 3 Risk of bias assessment for RCTs and pre-post studies

\begin{tabular}{|c|c|c|c|c|c|c|}
\hline \multirow[b]{2}{*}{ Source } & \multicolumn{6}{|c|}{ Risk of bias (high, low, unclear) } \\
\hline & $\begin{array}{l}\text { Randomisation } \\
\text { sequence } \\
\text { allocation }\end{array}$ & Concealment & $\begin{array}{l}\text { Performance } \\
\text { bias }\end{array}$ & $\begin{array}{l}\text { Ascertainment } \\
\text { bias }\end{array}$ & $\begin{array}{l}\text { Incomplete } \\
\text { outcome data }\end{array}$ & $\begin{array}{l}\text { Selective } \\
\text { reporting }\end{array}$ \\
\hline \multicolumn{7}{|l|}{ RCTs } \\
\hline Campbell et $a /^{33}$ & Low & Low & Unclear & Unclear & Low & Unclear \\
\hline Chen et $\mathrm{a} \mathrm{l}^{39}$ & Unclear & Unclear & Low & Low & Low & Unclear \\
\hline Gleeson et $\left.a\right|^{25}$ & Low & Low & Unclear & Low & Low & Unclear \\
\hline Hackney et al ${ }^{32}$ & High & High & High & Unclear & Low & Unclear \\
\hline Jeter et $a l^{42}$ & Low & Low & High & Low & Low & High \\
\hline Kovacs et $a l^{30}$ & Low & Low & Unclear & Low & Low & Unclear \\
\hline $\begin{array}{l}\text { Ackley-Holbrook et } \\
a l^{31}\end{array}$ & - & - & - & - & Low & Unclear \\
\hline Jeter et $a l^{41}$ & - & - & - & - & High & Unclear \\
\hline Kingston ${ }^{37}$ & - & - & - & - & Unclear & Unclear \\
\hline Larsson $^{43}$ & - & - & - & - & Low & Unclear \\
\hline Miszko et al ${ }^{40}$ & - & - & - & - & Unclear & Unclear \\
\hline Ponchillia et $\left.a\right|^{27}$ & - & - & - & - & Low & Unclear \\
\hline Salari $^{28}$ & - & - & - & - & Unclear & Unclear \\
\hline Surakka ${ }^{35}$ & - & - & - & - & Unclear & Unclear \\
\hline
\end{tabular}

*Not applicable.

$\mathrm{RCT}$, randomised controlled trials.

results in a meta-analysis, the evidence for intervention effectiveness is less clear.

This review identified 17 intervention studies, which represents a considerably small evidence base, particularly in contrast to the size of the problems related to physical inactivity, health conditions and challenges faced by many people with visual impairment. Several characteristics of existing studies have also limited the internal and external validity. First, most studies included very small sample sizes with four presenting descriptive analysis only (did not report inferential statistics) and two reporting results at an individual, rather than group level. Second, there was a substantial imbalance in both gender ( $70 \%$ female participants) and age with all but two studies focused on individuals aged older than 45 years. However, overrepresentation of female participants is common in health interventions and people aged 50 years and older represent $65 \%$ of all visually impaired persons worldwide (and $82 \%$ of blind persons) ${ }^{44}$ Third, more than three-quarters of the studies ran for 12 weeks or less, limiting the ability to assess maintenance of changes observed and effects on outcomes that may take time to change. For example, of the six studies assessing psychological well-being and mental health outcomes, none reported statistically significant improvements. However, promising results were observed in measures of functional capacity, such as mobility and fitness, and balance, even in a relatively short time period of 5-6 weeks. ${ }^{36}$ To address these issues future interventions should, where possible, employ an RCT study design with a priori calculation of participants needed to ensure the study is powered for more robust statistical analysis. If feasible, a longer duration would also be of benefit, particularly in establishing the impact of the interventions on mental health outcomes. Also, a targeted approach to include males and those aged 18-45 years may be appropriate to address the underrepresentation of these demographics in the current literature.

A wide range of physical activities were used by the intervention studies, most of which focused on lowintensity physical activities, such as yoga and tai chi, with a strong emphasis on improving balance and stability. Only one study incorporated higher intensity activity in the form of aerobics with 'many high-impact (bouncing and jumping) movements'. ${ }^{27}$ However, this study was also the smallest with only three participants, so it is hard to determine its effects. Although the benefits of yoga, tai chi and other low-intensity activities have been documented, ${ }^{45-47}$ moderate and vigorous physical activity have further health 
benefits and are generally the primary focus of global physical activity guidelines. ${ }^{5}$ The current CDC guidelines recommend a minimum of 150 min per week of moderate activity per week (or $75 \mathrm{~min}$ of vigorous activity) ${ }^{5}$ with the evidence on light intensity physical activity not yet conclusive for informing guidelines. ${ }^{1}$ Although these guidelines highlight a dose-response relationship whereby more health benefits are gained with an increase in moderate intensity physical activity undertaken, even small increases in physiological capacity/physical activity provide significant reduction in mortality risk. ${ }^{48} 49$ This suggests that future physical activity interventions among those with visual impairment should consider incorporating some physical activity at a moderate intensity or higher, even if only in small doses in older individuals. Although on the surface visual impairment may appear to be a barrier to undertaking moderate or vigorous physical activity, the existence of numerous sports for visually impaired athletes at Paralympic level would suggest otherwise. ${ }^{50}$ Even though few individuals reach this height of athletic ability, it is evident that visually impaired individuals can perform higher intensity activities, evidenced by the feasibility of physical activity trials with walking and aerobics as the intervention. ${ }^{27} 31$ Therefore, development of interventions for moderate and/or vigorous physical activity could form a focus in future work. Moreover, given the increased risk of poorer physical and mental health for those who are visually impaired, ${ }^{13} 51$ it is important to ensure they are given opportunities to undertake physical activity at higher intensities in order to garner the further health benefits.

In terms of delivery mode, studies were predominately group-based, with 9 of 16 (delivery mode unknown in one study) purely delivered in a face-to-face manner, and a further four involving group classes with additional selfdirected practice at home. The remaining three studies were of a more self-directed nature with varying levels of investigator involvement including five home visits throughout the yearlong study, ${ }^{33}$ five home visits (from occupational therapist or peer mentor) and two phone calls over the 6-month duration, ${ }^{34}$ and a single orientation session followed by self-directed activity. ${ }^{31}$ Further intervention studies could compare the effectiveness of groupbased and self-directed trials to determine the possibility of reducing investigator burden in delivery by increasing self-directed options where possible. This would have the added benefit of incorporating capacity building into the intervention and enabling participants to continue in their new habits postintervention. Alternatively, the use of already existing programmes such as regular community dance classes could be examined as a means of increasing the likelihood of maintaining changes to physical activity behaviours following the intervention. To our knowledge, none of the group-based interventions identified allowed participants to continue in the physical activity following the study as they were all conducted for research purposes only. However, given the habitual nature physical activity, it is possible that highly motivated individuals may have continued to practice in their own homes following instruction during the intervention.

\section{Strengths and limitations}

The strengths of this systematic review include incorporating both qualitative and quantitative evidence synthesis, following AMSTAR2 and PRISMA guidelines, and not restricting literature search by publication language and dates. Limitations relate to the studies identified, rather than the review process itself. The studies identified were generally small with diverse study designs and outcomes measured. This made it challenging to determine the effectiveness of the interventions and to identify the aspects that should be retained in subsequent studies. Most studies lasted for a short period of time without intended examination for longer-term maintenance. Finally, the quality assessment showed a high risk of bias across the papers in several categories.

\section{CONCLUSION}

Given the higher risk of developing non-communicable diseases for those with visual impairment, it is imperative that sufficient physical activity is undertaken by these individuals to ensure that they benefit from the positive health outcomes. This systematic review illustrates that physical activity interventions in individuals with visual impairment can have beneficial results, particularly in physical measures such as mobility and balance. However, when performing a meta-analysis of RCTs, the evidence for effectiveness is less clear. More high-quality research needs to be conducted in larger groups, with a focus on specific age groups and over longer periods of time to enable the optimisation of further interventions. Additionally, future studies need to incorporate interventions that equip the participants with skills and confidence to sustain their new physical activity behaviours postintervention. Finally, more research is required into the feasibility of interventions that address the need for moderate and vigorous physical activity, which unlock even more health benefits compared with the low-intensity activities reported in this systematic review.

\section{Author affiliations}

${ }^{1}$ Charles Perkins Centre, The University of Sydney, Camperdown, New South Wales, Australia

${ }^{2}$ Prevention Research Collaboration, The University of Sydney School of Public Health, Camperdown, New South Wales, Australia

${ }^{3}$ Physical Activity and Health, Western Sydney University School of Science and Health, Campbelltown, New South Wales, Australia

${ }^{4}$ Department of Public Health and Faculty of Medicine, Universitas Udayana, Bukit Jimbaran, Bali, Indonesia

${ }^{5}$ Sydney Dental School, The University of Sydney, Camperdown, New South Wales, Australia

${ }^{6}$ Faculty of Health Sciences, The University of Sydney, Cumberland, New South Wales, Australia

Acknowledgements The authors wish to thank Dr Javad Koohsari, from Waseda University, Japan, for helping with translation and data extraction from a study published in Farsi. 
Contributors JS, DM, MA, KE and DD were involved in study design. JS and MA performed the systematic search. JS, DM, PASA, KE and DD made significant contributions to the analysis and/or interpretation of the data. JS, DM, PASA, MA, KE and DD all contributed to drafting and revising the work.

Funding The work was supported by a Sydney Research Accelerator (SOAR) prize awarded to Ding. Ding is funded by a National Heart Foundation Future Leader Fellowship.

Competing interests None declared.

Patient consent for publication Not required.

Provenance and peer review Not commissioned; externally peer reviewed.

Data availability statement All data relevant to the study are included in the article or uploaded as supplementary information.

Open access This is an open access article distributed in accordance with the Creative Commons Attribution Non Commercial (CC BY-NC 4.0) license, which permits others to distribute, remix, adapt, build upon this work non-commercially, and license their derivative works on different terms, provided the original work is properly cited, appropriate credit is given, any changes made indicated, and the use is non-commercial. See: http://creativecommons.org/licenses/by-nc/4.0/.

\section{ORCID iDs}

Joanna Sweeting http://orcid.org/0000-0001-9497-601X

Dafna Merom http://orcid.org/0000-0001-6459-8628

Putu Ayu Swandewi Astuti http://orcid.org/0000-0002-4723-212X

Kate Edwards http://orcid.org/0000-0003-3429-5989

Ding Ding http://orcid.org/0000-0001-9850-9224

\section{REFERENCES}

12018 Physical Activity Guidelines Advisory Committee. 2018 physical activity guidelines Advisory Committee scientific report. Washington, DC, 2018. https://health.gov/paguidelines/second-edition/report/pdf/ pag_advisory_committee_report.pdf

2 Lee I-M, Shiroma EJ, Lobelo F, et al. Effect of physical inactivity on major non-communicable diseases worldwide: an analysis of burden of disease and life expectancy. The Lancet 2012;380:219-29.

3 Chekroud SR, Gueorguieva R, Zheutlin AB, et al. Association between physical exercise and mental health in 1.2 million individuals in the USA between 2011 and 2015: a cross-sectional study. Lancet Psychiatry 2018;5:739-46.

4 Penedo FJ, Dahn JR. Exercise and well-being: a review of mental and physical health benefits associated with physical activity. Curr Opin Psychiatry 2005;18:189-93.

5 U.S. Department of Health and Human Services. Physical activity guidelines for Americans. 2nd edn. Washington, DC: U. S. Department of Health and Human Services, 2018. https://health.gov/ paguidelines/second-edition/pdf/Physical_Activity_Guidelines_2nd_ edition.pdf

6 Guthold R, Stevens GA, Riley LM, et al. Worldwide trends in insufficient physical activity from 2001 to 2016: a pooled analysis of 358 population-based surveys with 1.9 million participants. Lancet Glob Health 2018;6:e1077-86.

7 U.S. Department of Health and Human Services. Increasing physical activity among adults with disabilities, 2018. Available: https://www. cdc.gov/ncbddd/disabilityandhealth/pa.html [Accessed Apr 2019].

8 Okoro CA, Hollis ND, Cyrus AC, et al. Prevalence of disabilities and health care access by disability status and type among adults United States, 2016. MMWR Morb Mortal Wkly Rep 2018;67:882-7.

9 World Health Organization. Blindness and visual impairment, 2018. Available: https://www.who.int/news-room/fact-sheets/detail/ blindness-and-visual-impairment [Accessed Mar 2019].

10 Langelaan M, de Boer MR, van Nispen RMA, et al. Impact of visual impairment on quality of life: a comparison with quality of life in the general population and with other chronic conditions. Ophthalmic Epidemiol 2007;14:119-26.

11 Vu HTV, Keeffe JE, McCarty CA, et al. Impact of unilateral and bilateral vision loss on quality of life. Br J Ophthalmol 2005;89:360-3.

12 Zhang X, Bullard KM, Cotch MF, et al. Association between depression and functional vision loss in persons 20 years of age or older in the United States, NHANES 2005-2008. JAMA Ophthalmo 2013;131:573-81.

13 McCarty CA, Nanjan MB, Taylor HR. Vision impairment predicts 5 year mortality. Br J Ophthalmol 2001;85:322-6.

14 Taylor HR, Katala S, Muñoz B, et al. Increase in mortality associated with blindness in rural Africa. Bull World Health Organ 1991;69:335-8.
15 Ramrattan RSet al. Prevalence and causes of visual field loss in the elderly and associations with impairment in daily functioning. Arch Ophthal 2001;119:1788-94

16 Nguyen AM, Arora KS, Swenor BK, et al. Physical activity restriction in age-related eye disease: a cross-sectional study exploring fear of falling as a potential mediator. BMC Geriatr 2015;15:64.

17 van Landingham SW, Willis JR, Vitale S, et al. Visual field loss and accelerometer-measured physical activity in the United States. Ophthalmology 2012;119:2486-92.

18 Willis JR, Jefferys JL, Vitale S, et al. Visual impairment, uncorrected refractive error, and accelerometer-defined physical activity in the United States. Arch Ophthalmol 2012;130:329-35.

19 Kirchner CE, Gerber EG, Smith BC, et al. Community barriers to physical activity for people with visual or motor impairments. Am J Prev Med 2008;34:349-52.

20 Krahn GL, Walker DK, Correa-De-Araujo R. Persons with disabilities as an unrecognized health disparity population. Am J Public Health 2015;105:S198-206.

21 Piva da Cunha Furtado OL, Allums-Featherston K, Lieberman LJ, et al. Physical activity interventions for children and youth with visual impairments. Adapt Phys Activ Q 2015;32:156-76.

22 Moher D, Liberati A, Tetzlaff J, et al. Preferred reporting items for systematic reviews and meta-analyses: the PRISMA statement 2009

23 Shea BJ, Reeves BC, Wells G, et al. AMSTAR 2: a critical appraisal tool for systematic reviews that include randomised or nonrandomised studies of healthcare interventions, or both. BMJ 2017;358:j4008.

24 Higgins JPT, Green S. Cochrane Handbook for systematic reviews of interventions. The Cochrane collaboration. Available: www. handbook.cochrane.org [Accessed May 2019].

25 Gleeson M, Sherrington C, Lo S, et al. Impact of the Alexander technique on well-being: a randomised controlled trial involving older adults with visual impairment. Clin Exp Optom 2017;100:633-41.

26 Gleeson M, Sherrington C, Lo S, et al. Can the Alexander technique improve balance and mobility in older adults with visual impairments? A randomized controlled trial. Clin Rehabil 2015;29:244-60

27 Ponchillia SV, Powell LL, Felski KA, et al. The effectiveness of aerobic exercise instruction for totally blind women. J Visual Impair Blin 1992;86:174-7

28 Salari A, Sahebalzamani M, Daneshmandi $H$. The effects of core stability training program on balance in blind female athletes. Journal of Kerman University of Medical Sciences 2013;20:585-95.

29 Cheung KKW, Au KY, Lam WWS, et al. Effects of a structured exercise programme on functional balance in visually impaired elderly living in a residential setting. Hong Kong Physiotherapy Journal 2008;26:45-50.

30 Kovács Éva, Tóth $\mathrm{K}$, Dénes L, et al. Effects of exercise programs on balance in older women with age-related visual problems: a pilot study. Arch Gerontol Geriatr 2012;55:446-52.

31 Ackley-Holbrook E, Kang M, Morgan DW. Development and evaluation of the walk for health program: a physical activity intervention for adults with visual impairments. J Vis Impair Blind 2016:110:103-14.

32 Hackney ME, Hall CD, Echt KV, et al. Multimodal exercise benefits mobility in older adults with visual impairment: a preliminary study. $J$ Aging Phys Act 2015;23:630-9.

33 Campbell AJ, Robertson MC, Grow SJL, et al. Randomised controlled trial of prevention of falls in people aged $\geq 75$ with severe visual impairment: the VIP trial. BMJ 2005;331:817.

34 Waterman $\mathrm{H}$, Ballinger $\mathrm{C}$, Brundle $\mathrm{C}$, et al. A feasibility study to prevent falls in older people who are sight impaired: the VIP2UK randomised controlled trial. Trials 2016;17:464

35 Surakka A, Kivela T. Motivating visually impaired and deaf-blind people to perform regular physical exercises. Br J Vis Impair 2008;26:255-68

36 Surakka A, Kivela T. The effect of a physical training programme on flexibility of upper body and trunk in visually impaired and deaf-blind persons. Eur J Adapt Phys Act 2011;4:7-21.

37 Kingston JT, Impairment V. Visual impairment and falls: outcomes of two fall risk assessments after a Four-Week fall prevention program. $J$ Vis Impair Blind 2018;112:411-5.

38 Tennstedt S, Howland J, Lachman M, et al. A randomized, controlled trial of a group intervention to reduce fear of falling and associated activity restriction in older adults. J Gerontol B Psychol Sci Soc Sci 1998;53B:P384-92.

39 Chen EW, Fu ASN, Chan KM, et al. The effects of tai chi on the balance control of elderly persons with visual impairment: a randomised clinical trial. Age Ageing 2012;41:254-9.

40 Miszko TA, Ramsey VK, Blasch BB. Tai chi for people with visual impairments: a pilot study. J Vis Impair Blind 2004;98:5-13. 
41 Jeter PE, Dagnelie G, Khalsa SBS, et al. Yoga for persons with severe visual impairment: a feasibility study. Alternative Medicine Studies 2012;2:e5:5.

42 Jeter PE, Haaz Moonaz S, Bittner AK, et al. Ashtanga-Based yoga therapy increases the sensory contribution to postural stability in Visually-Impaired persons at risk for falls as measured by the Wii balance board: a pilot randomized controlled trial. PLoS One 2015;10:e0129646.

43 Larsson L, Frändin K, Awareness B. Body awareness and DanceBased training for persons with acquired Blindness-Effects on balance and gait speed. Vis Impair Res 2006;8:25-40.

44 Pascolini D, Mariotti SP. Global estimates of visual impairment: 2010. Br J Ophthalmol 2012;96:614-8.

45 Chu P, Gotink RA, Yeh GY, et al. The effectiveness of yoga in modifying risk factors for cardiovascular disease and metabolic syndrome: a systematic review and meta-analysis of randomized controlled trials. Eur J Prev Cardiol 2016;23:291-307.
46 Ross $\mathrm{A}$, Thomas $\mathrm{S}$. The health benefits of yoga and exercise: a review of comparison studies. J Altern Complement Med 2010;16:3-12.

47 Jahnke R, Larkey L, Rogers C, et al. A comprehensive review of health benefits of qigong and tai chi. Am J Health Promot 2010;24:e1-25.

48 Arem $\mathrm{H}$, Moore SC, Patel A, et al. Leisure time physical activity and mortality: a detailed pooled analysis of the dose-response relationship. JAMA Intern Med 2015;175:959-67.

49 Mandsager K, Harb S, Cremer P, et al. Association of cardiorespiratory fitness with long-term mortality among adults undergoing exercise treadmill testing. JAMA Netw Open 2018;1:e183605.

50 Paralympics Australia. Vision classification, 2019. Available: https:// www.paralympic.org.au/vision-classification/ [Accessed Apr 2019].

51 Court H, McLean G, Guthrie B, et al. Visual impairment is associated with physical and mental comorbidities in older adults: a crosssectional study. BMC Med 2014;12. 